\title{
LA CULTURA PRÓXIMA COMO FUENTE DE CONTENIDOS SIGNIFICATIVOS DE UN DISEÑO CURRICULAR BASADO EN LA COMPRENSIÓN DE CONCEPTOS
}

\author{
JULIO VERA VILA \\ Universidad de Málaga
}

Desde la primera infancia, el niño aprende a relacionarse con la realidad que le rodea a través de las interpretaciones con carga valorativa que le proporcionan la tradición y las personas más próximas en su microcontexto, fundamentalmente el familiar, pero también, y cada vez más, el vecinal, el local, etc. Este conjunto de claves que constituyen el primer código con el que desvelar la carga semántica de las cosas, de las personas, de los hechos, etc, se amplía y enriquece a medida que crece y acumula más experiencias relacionales.

Esta inmersión del niño en la cultura de su grupo (Castillejo, 1985, Cfr. p. 10) y su relación espontánea con el entorno próximo que le rodea, favorece el desarrollo de un conocimiento vulgar, empírico, que le va proporcionando una imagen relativamente vaga, no justificada científicamente, de la realidad, a la vez que le pertrecha de un bagaje de creencias que no son producto de su razonamiento sino una codificación social, común y compartida de la realidad. En Ortega, las creencias,son un tipo particular de vigencias; justamente aquellas que se refieren a la interpretación de la realidad (Marías, 1972, Cfr. p. 125) y que, debido al efecto de la inmersión cultural, actúan en nosotros de un modo inconsciente, es decir, no son pensadas. Y no lo son porque no son un sistema teórico sino vital. "La creencia es certidumbre en que nos encontramos sin saber cómo ni por dónde hemos entrado en ella". (Ortega, 1976, p. 58)

La educación puede ayudar al sujeto a dar el paso por el que emergiendo del propio grupo con la ayuda de los símbolos que en él ha ido adquiriendo, y que han constituido el material informativo a procesar, amplía el horizonte de sus expectativas, y la propia cultura que hasta ese momento era toda la realidad, se comprende como un elemento más de una clase de categorías de pensamiento. La CULTURA es comprendida, entonces, como un concepto que engloba una categoría de objetos de los que forma parte 
la propia cultura de uno. Las seguridades incuestionadas en que se desenvolvía en un primer momento comienzan a ser discutidas y se abre el camino para la duda. "La duda es algo que pasa de pronto al que antes tenía una fe o creencia, en la cual se hallaba sin más y desde siempre" (Ortega, p. 57). La misma creencia que le había servido como clave interpretativa de la realidad, toma ante él la apariencia de ser una parte más de ésta última, convirtiéndose, entonces, en contenido del pensamiento, en una idea en términos orteguianos. El cambio operado en la estructura cognitiva del sujeto le permite darse cuenta de que ha de optar por un modo concreto de ser hombre dentro de las posibilidades del momento que le ha tocado vivir y dentro de las limitaciones naturales y culturales, diacrónicas y sincrónicas.

Sugerimos aquí, que la reflexión anterior puede servir como criterio pertinente para el diseño de modelos curriculares, sobre todo, en lo relacionado con el problema de la selección y organización de los contenidos, cuando se plantea como objetivo el que el alumno asimile, significativamente, la cultura y el conocimiento de la comunidad en la que vive. Creo que el currículum educativo, sea escolar o no, debe estar elaborado de tal forma que ayude al educando a comprender su situación dentro del microtexto social al que pertenece, así como el lugar de su propio grupo social de origen dentro de la sociedad plural en la que vivimos. El potencial cultural del grupo social de origen, es decir, aquel que le ha servido como microcontexto de sus primeros aprendizajes y que está constituido por conocimientos, modelos, normas de conducta, puestas en acción, etc. (Castillejo, 1981 a, Cfr. p. 102), se constituiría en una rica fuente de contenidos significativos, que una vez asimilados y comprendidos se convertirían en facilitadores de la transferencia ${ }^{1}$ de esos aprendizajes a otros contextos culturales más amplios, con los que comparte regularidades que se repiten por ser todos ellos sistemas socioculturales de relaciones humanas.

Para este propósito parece interesante adoptar la distinción entre problemas curriculares y aquellos que son propios de la instrucción (Novak, 1982, Cfr. p. 122). En el primer caso estamos ante la exigencia de encontrar, primero, aquellas regularidades y generalizaciones comunes a una misma clase de objetos ${ }^{2}$ que permite conceptualizarlos como elementos singulares de un conjunto y, posteriormente, en un nivel más alto de abstracción encontrar los isomorfismos a los que transferir, lo aprendido en la propia

1. Respecto a los procesos de transferencia de los aprendizajes, Ausubel afirma que " [...] incorporar a la estructura cognitiva ideas inclusivas, claras, estables e integradoras es la manera más eficaz de fomentar la transferencia." (AUSUBEL, 1976, p. 192).

2. Empleo del término "objeto" en el sentido más amplio, no para referirme exclusivamente a lo concreto o a lo físico, sino en general a cualquier ente, a aquello que existe o puede existir, a aquello que es pensado o que puede serlo. 
cultura, a otras culturas, con lo que, como ya se dijo anteriormente, el microcontexto que en un primer momento se confundía con la realidad misma, pasa a conceptualizarse también como un elemento de un conjunto: el conjunto de las formas de vida humana en comunidad. Seguramente, de esta forma es más accesible a la comprensión del alumno, no sólo el legado histórico, sino también, y ello es muy importante, los procesos heterogéneos de su producción. En el segundo caso (problemas de instrucción) se trata de utilizar los objetos, símbolos, códigos, medios, técnicas y referentes, no de cualquier cultura, sino aquella que, a nuestro juicio, permite eliminar mayor cantidad de arbitrariedad, ruido y entropía, es decir, la cultura próxima al sujeto. Este, desde luego, no será el único criterio relevante, ni para el diseño curricular, ni para la planificación de la instrucción, pero parece lo suficientemente consistente como para tenerlo en cuenta.

Si observamos, por tomar un ejemplo, el diseño curricular e instructivo de los textos escolares encontraremos que toman en consideración criterios epistemológicos, el nivel de conocimientos científicos actuales acerca de la realidad, la lógica interna de las diferentes materias de estudio. En ocasiones podemos encontrar también esfuerzos interdisciplinares, pero por diferentes razones, entre ellas las comerciales, ni parten de la realidad más próxima al sujeto destinatario, ni toman en consideración sus estructuras cognitivas, ni los posibles conceptos inclusores (sets de aprendizaje anteriores en la terminología de Castillejo), ni sus claves interpretativas. Lo cual conduce a aprender conceptos y principios generales con ejemplificaciones que todavía no forman parte de su repertorio de información procesada significativamente, por lo que ha de almacenarse de forma arbitraria y aislada, agotándose su funcionalidad de servir para pasar, favorablemente, la evaluación escolar. ¿Qué ha ocurrido? Que entre los inputs formalizados de los libros de texto, así como entre las formas regladas de su procesamiento, y los inputs informales o formales de microcontexto hay una disonancia, que será mayor o menor en función de origen sociocultural de los sujetos. De ahí que aunque dos personas sean expuestas a un mismo input, el valor informativo de éste variará al estar afectado por la forma particular en que es procesado por cada una de ellas. en efecto, desde la perspectiva cognitiva, y a pesar de las limitaciones impuestas por la falta de evidencia empírica, se parte del supuesto de que entre el input y el output hay procesos mediacionales activos que son información en mensaje, mediante un complejo sistema en el que la información es recibida, transformada, acumulada, recuperada y utilizada en base a experiencias anteriores (MAHONEY, 1974, Cfr. p. 415-416). Entre uno y otro, hay pues, un proceso previo de codificación con una experiencia afectiva asociada.

Para la elaboración de modelos curriculares basados en tales propuestas, me parecen relevantes, como de alguna manera ha quedado ya insinua- 
do, dos soportes teóricos: los enfoques sistémico-cibernéticos y las teorías cognitivas del aprendizaje, entre otras razones, porque posibilitan niveles altos de congruencia entre los criterios de selección y organización de los contenidos, los procesos subjetivos de comprensión y la secuencialización de la enseñanza.

\section{LA SELECCION Y ORGANIZACION DE LOS CONTENIDOS}

Según la Teoría General de Sistemas, la realidad es un conjunto de totalidades organizadas, cuyos elementos funcionan dentro de una red de intereacciones con los demás elementos, de tal modo, que la modificación de uno sólo de ellos, repercute en la configuración del conjunto. Gracias a los isomorfismos observados en la realidad, es decir a las similitudes estructurales entre los diferentes sistemas y subsistemas, es posible formular principios generales aplicables a todos ellos, independientemente de la naturaleza específica de cada uno de ellos. Ello conduce a la negación de la compartimentalización del saber en ciencias particulares como si proviniera del estudio de realidades diversas e inconexas, y a la defensa, por el contrario de la interdisciplinariedad como metodología adecuada a una realidad única y sistémica (Colom, 1982, cfr. pp. 121-126). En definitiva, el objeto consistiría en formalizar esquemas conceptuales aplicables a toda realidad que pueda ser considerada como un sistema, para generar principios, leyes y modelos transferibles de unos campos científicos a otros (Colom, cfr. p. 131).

Hay una gran similitud entre este planteamiento y el trabajo que ha de realizar el sujeto cuando quiere transferir su aprendizaje de un campo a otro o cuando busca las regularidades compartidas por varios casos particulares. En ambas situaciones se trata de comprender, de codificar parcelas de la realidad de forma inteligible. Así como la T.G.S. busca la comunicación interdisciplinar entre las ciencias para representar al mundo como un conjunto de fenómenos interrelacionados, como un conjunto de todos organizados, el sujeto para comprender, establece conexiones y organizaciones con diferentes grados de generalidad, y su pensamiento trabaja con un todo de conceptos y secuencias lógicas interrelacionadas. Por ello creo que todo modelo de diseño curricular que parta de la consideración sistemica de la realidad para la organización de los contenidos, se ajusta mejor al objetivo de favorecer al comprensión en los sujetos, que los modelos organizados por materias sin nexos de unión.

\section{EL CONCEPTO DE COMPRENSION Y EL APRENDIZAJE SIGNIFICATIVO.}

Podemos definir la comprensión como "'la capacidad para relacionar 
lo que se ha de comprender con alguna estructura más amplia, más o menos determinada', o 'capacidad para ligar lo que se ha de comprender con lo que ya se conoce o comprende"' (Woods y Barrow, 1978, p. 51). Es un proceso que conduce al conocimiento del por qué de las cosas a través de la relación entre conceptos, razonamientos, o secuencias lógicas, donde lo nuevo es encajado en el conocimiento anterior, previamente ordenado, que a su vez, conducirá a su reordenamiento de la anterior. Esto es válido tanto para el significante como para el significado. En cuanto al significante consistirá en la capacidad para vincular los signos, soportes de una información, con el vocabulario, o caudal de signos en general, que ya se domina. en cuanto al significado, se comprende cuando se es capaz de encajarlo en una estructura más amplía, donde se engarza un concepto con otros, una secuencia lógica con otras o un discurso con otros, en niveles de creciente abstracción.

El sujeto en su interacción con el medio, va a topar con datos, objetos, hechos, que ha de ser capaz de identificar como casos concretos referidos a principios generales, de los cuales no son sino manifestaciones o concreciones. La comprensión requiere la posibilidad de ir de los elementos singulares a los principios generales, como ocurre en los primeros aprendizajes, pero también, la disposición para aplicar lo general a lo particular, lo abstracto a lo concreto.

Normalmente el propósito de los educadores no es que los alumnos repitan como loros una información, sino que sean capaces de operar inteligentemente con ella. ¿Pero cómo saber que ha comprendido? ¿Cómo ayudar a comprender? ¿Cómo determinar los criterios fundamentales en la selección de los contenidos para facilitar la comprensión? Desde luego, antes de cualquier tentativa de dilucidar nada por medio de una investigación empírica, y previo a todo diseño curricular o instructivo abocado al desarrollo de la comprensión, es preciso definirla con la mayor exactitud posible.

En todo campo de conocimientos hay unas prioridades lógicas, de tal modo, que los niveles inferiores preceden y justifican a los superiores (HIRST, 1969, Cfr. p. 88-92). Además, los conceptos se hallan relacionados formando redes, y hay reglas que gobiernan su utilización de tal manera que quede reducida al mínimo su ambigüedad semántica ${ }^{3}$. Comprender un todo significa entender sus componentes más simples en sus relaciones recíprocas. Tener el concepto de algo ${ }^{4}$ es conocer el principio al que se invoca cuando

3. Acerca de problemas tales como la ambigüedad contextual y la anemia semántica, directamente relacionados en el tema que nos ocupa, es importante el libro de Esteve (1979). Yo he utilizado la expresión "ambigüedad semántica" en el mismo sentido que él emplea, explicando a Black, el de "ambigüedad contextual".

4. Henderson, ayuda, no poco a la discusión rigurosa de este tema. Particularmente toma como punto de partida el hecho de que todo concepto supone una cuádruple relación entre: términos (significantes), personas (que comparten los conceptos), contextos (de la relación) y significado (HENDERSON, 1.972, Cfr.. pp. 85-92). 
se dice que ciertas cosas son de una clase determinada. Tenemos un concepto cuando podemos señalar las cosas que engloba y encontrar las razones que justifican su pertenencia a un conjunto.

Las cosas que atañen inmediata y originalmente al sujeto, son primeramente particulares y concretas. En la medida en que la experiencia se ensancha van apareciendo diferentes grados de abstracción y con ella una percepción de la realidad más interpersonal, abstracta y general.

Así pues, una educación que sirva para mejorar la comprensión humana, puede conseguirse a través de un intento deliberado por mejorar el número y la calidad de los conceptos que tienen las personas. La ideas de Ausubel han sido calificadas, acertadamente, como una psicología en la que la comprensión representa un papel fundamental dentro del aprendizaje, llegando a afirmarse que el aprendizaje de conceptos es la función primordial de la enseñanza (NOVAK, Cfr. p. 59).

El aprendizaje significativo se produce cuando una nueva información entra en relación con algún elemento relevante, ya existente en la estructura cognitiva del sujeto (AUSUBEL, 1976, Cfr. pp. 134-135). En ésta última se supone la existencia de un conjunto de conceptos previos, asimilados con anterioridad, relacionados dinámicamente de tal forma que siendo actualizados en el momento oportuno, le permiten al sujeto interactuar con el mundo, satisfacer necesidades, solucionar problemas, elaborar una representación de la realidad y operar de acuerdo a ella.

Como he venido repitiendo, los primeros inclusores los elaboramos por abstracción de los elementos y objetos culturales de nuestro microcontexto social. Esta inmersión dificultará durante algún tiempo, pero también facilitará, a la larga, la contemplación de su vida relacional próxima como un caso particular de cultura. Mientras esta etapa perdure, habrá una dificultad grande en comprender otras formas de vida diferentes a la propia. Y este escollo no es difícil que nos aparezca alguna vez o que esté instalado, en ciertas personas, como una actitud consistente (rasgos) (CASTILLEJO, 1981a, Cfr. pp. 114-120), manifestándose en conductas intolerantes hacia quienes piensan, sienten o se comportan de una forma distinta a la de uno mismo.

\section{LA SECUENCIALIZACION DE LOS CONTENIDOS EN LA ENSEÑANZA}

Hemos dicho que para dominar un campo cualquiera del saber, es necesario adquirir la capacidad de utilizar los conceptos, en él implicados, de acuerdo con las reglas que gobiernan las relaciones de los unos con los otros para construir proposiciones legales y razonamientos válidos. Ahora bien, de ello no se deduce, necesariamente, que la enseñanza/aprendizaje haya 
de seguir un orden temporal necesario, porque eso significaría haber contemplado los aspectos lógicos a costa de olvidar lo psicológico 5 . La enseñanza debe implicar una secuencialización, un ordenamiento lógico, pero de ahí no se concluye que deba ser siempre el mismo, ni tampoco igual para todos.

Veamos dos ejemplos analógicos, muy recurridos en estos casos, y utilizados por Hirst. En un rompecabezas, cada pieza está relacionada con otras varias con las que encaja. De igual modo, las colaterales se relacionan con las vecinas y así sucesivamente. Sin embargo para completarlo, podemos comenzar por cualquier pieza, con la única condición de respetar las reglas lógicas por las que unas piezas encajan con otras. Mi propuesta vendría a significar que mientras el sujeto permanece inmerso en su microcontexto es similar a cuando intenta construir el rompecabezas, donde las piezas representan elementos de su ámbito cultural próximo, algunas de las cuales ya son conocidas por él y funcionan como inclusores. Pero cuando emerge el rompecabezas pasa a ser comprendido como un caso particular de rompecabezas y desvelado en su mecánica interna, de tal forma que se comienza a estar en disposición, no sólo de construir otros rompecabezas, sino también de diseñarlos. Los otros rompecabezas representarían, en el símil, a otras culturas donde la mecánica es práctica la misma, aunque las piezas y lo que cada una significa en relación a las otras varía. Evidentemente esta no es más que una simplificación muy tosca de como ocurren en la realidad las cosas, pero confio en que nos pueda aclarar algo.

El otro ejemplo está basado en el ajedrez. En este juego, el movimiento y la importancia de cada pieza esta relacionado con la posición que ocupan las demás en cada jugada y en cada nueva partida. A veces un peón no tiene importancia, otras puede decidir un final. Ningún intento estratégico puede ser consistente si el desenvolvimiento táctico no sigue un plan escalonada en el que se contemplen el mayor número de variantes posible. Sin embargo, un mismo objetivo estratégico puede ser alcanzado por líneas de desarrollo del juego diferentes.

Podemos encontrar pistas para una buena solución al problema de la secuencia temporal del aprendizaje en el concepto de organizador previo de Ausubel ${ }^{6}$. En este sentido, la elección de uno u otro concepto para que

5. Kneller, se interroga acerca de la influencia que debieran ejercer respectivamente, la lógica y la psicología en la secuencialización del aprendizaje cuando, como es notorio, no todo puede aprenderse al mismo tiempo y en consecuencia, el contenido ha de ordenarse de alguna manera. (KNELLER, 1969, cfr. p. 65).

6. "La ventaja de construir deliberadamente un organizador especial para cada unidad nueva de material estriba en que sólo de esta manera puede el alumno aprovechar las ventajas de un clasificador que: a) le da una visión general del material más detallado antes de que se enfrente realmente con él, y b) le suministra elementos organizadores que son inclusivos y que tienen en cuenta más pertinente y eficazmente el contenido en particular de este material" (AUSUBEL, p. 179). 
cumpla la función de organizador previo, en cierto modo es arbitraria, ya que cualquier secuencia específica que propongamos, no será sino una de la casi infinita variedad que podríamos elegir (NOVAK, cfr. p. 83). La condición necesaria que se ha de dar es, una vez más, el que se encuentre la relación entre los conceptos y organizar la enseñanza de tal manera, que permita subir y bajar, clínica y no linealmente, por las jerarquías conceptuales a medida que una nueva información es introducida, a fin de volver a presentar la forma en que los conceptos se reordenan al integrarse los elementos recién incorporados. De este modo se procede hacia un aprendizaje supraordenado en el que los elementos más simples se integran en un todo más amplio e inclusivo. El organizador previo hace las veces de puente o enlace entre lo que el alumno ya sabe y lo que necesita saber. En cualquier caso, para elegir los organizadores previos, habrá que conocer lo que el alumno ya sabe (los inclusores de que dispone) y por otro lado, la forma en que se relacionan los conceptos a aprender.

Al igual que la consideración de la realidad como sistema, también el principio de la reconciliación integradora $a^{7}$ conduce al rechazo, hasta donde ello sea posible, de la compartimentalización de los temas y de los conceptos, en definitiva de la realidad. Lo cual parece razonable, ya que en la medida en que aceptemos a la realidad como una e indivisible, más cerca estaremos de lograr un conocimiento comprensivo e integrador de la misma.

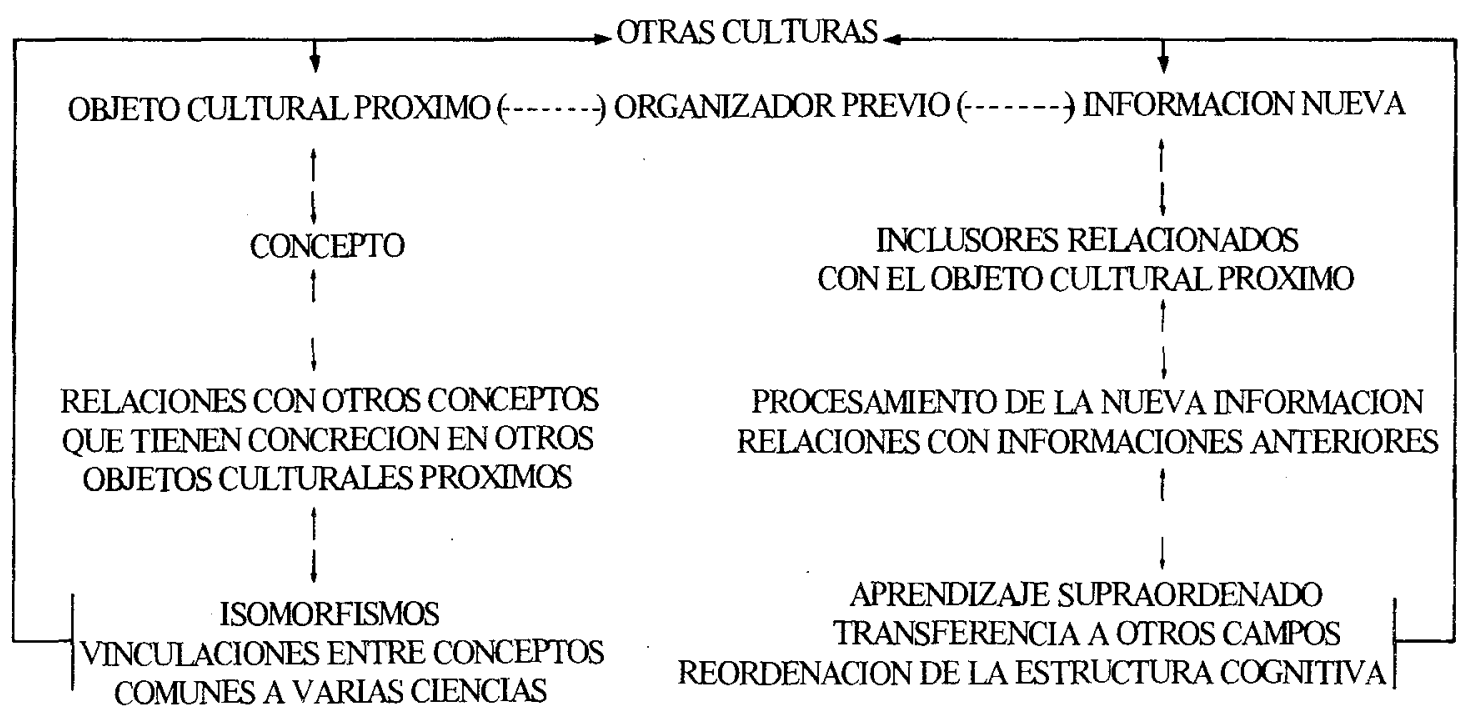

ESQUEMA Núm. 1

PARALELISMOS ENTRE LA REALIDAD SISTEMICA Y LA COMPRENSION GLOBALIZADA

7. "El principio de la reconciliación integradora al programar material de enseñanza puede describirse mejor como espíritu y enfoque opuestos a la difundida práctica entre los escritores de libros de texto, de departamentalizar y separar ideas o temas partibulares dentro de sus respectivos capítulos y subcapítulos. [...] Algunas de las consecuencias indeseables de este enfoque consiste en que se emplean términos múltiples para presentar conceptos de equivalencia intrínseca salvo en lo que respecta a la referencia de contexto [...]." (AUSUBEL, P. 186). 
Finalmente, en el esquema n. 2 he intentado elaborar, muy sintéticamente, los pasos que habría que seguir en la elaboración de un modelo de diseño curricular basado en las ideas anteriormente expuestas.

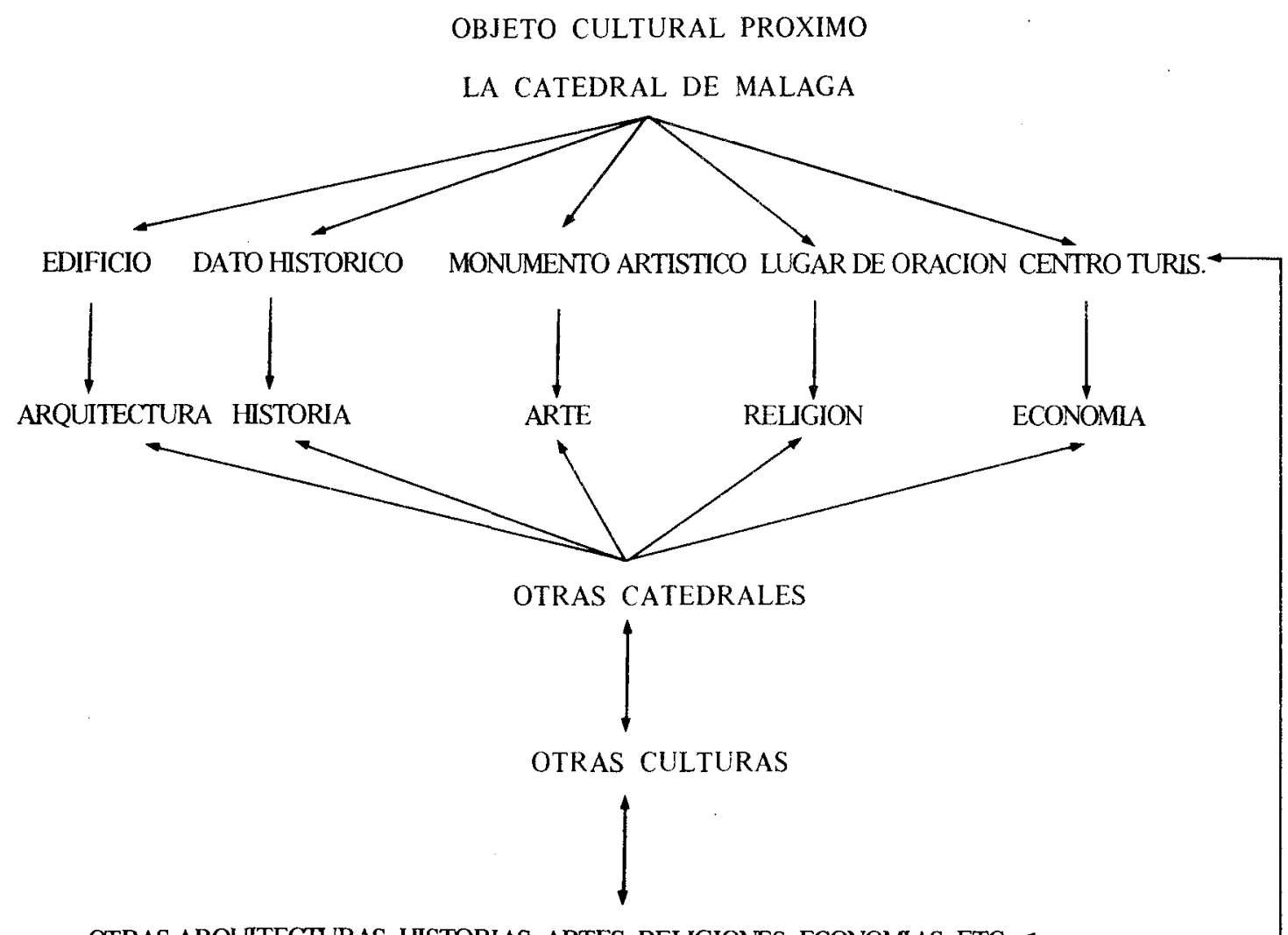

OTRAS ARQUTTECTURAS, HISTORIAS, ARTES, RELIGIONES, ECONOMIAS, ETC.

ESQUEMA Núm. 2:

LA CUlTURA PROXIMA COMO FUENTE DE CONTENIDOS SIGNIFICATIVOS, VINCULACION CON OTRAS CULTURAS. 


\section{BIBLIOGRAFIA}

AUSUBEL, D.P. (1976): Psicología educativa. Un punto de vista cognitivo Trillas, México.

BROUDY, H.S. (1976): "Dominio" en SMITH, B.O. y ENNIS, R.S.: Lenguaje y conceptos en educación, Ateneo, B. Aires.

CASTILLEJO BRULL, J.L. (1981a): "Modelo funcional del proceso educativo", en CASTILLEJO, J.L.; ESCAMEZ, J. y MARIN, R.: Teoría de la educación. Anaya, Madrid. (1981b): "El marco sociocultural de la educación", en CASTILLEJO, J.L.; ESCAMEZ, J. y MARIN, R.: Teoría de la educación. Anaya, Madrid.

(1985): "Educación y acción educativa" en VARIOS: Conceptos y propuestas II. Nau Llibres, Valencia.

(1986): Proceso educativo y construcción humana" en VARIOS: Conceptos y propuestas III. Nau Llibres, Valencia.

CLAUDIO PUERTO, A. (1986): Ambiente y educación: precisiones conceptuales y funcionales", en VARIOS: Conceptos y propuestas III. Nau Llibres, Valencia.

COLOM, A.J. (1982): Teoría y metateoría de la educación. Trillas, México.

(1983): “Concepto de educación ambiental", en VARIOS: Teoría de la educación I. Límites, Murcia.

ESTEVE ZARAZAGA, J.M. (1979): Lenguaje educativo y teorias pedagógicas. Anaya, Madrid.

HAMLYN, D.W. (1969) "Los aspectos lógico y psicológico del aprendizaje" en PETERS, R.S. (ed.) : El concepto de educación Paidós, B. Aires.

HENDERSON K.B. (1972): “Un modelo lógico para la conceptualización y otras actividades afines” en KOMISAR, B.P. Y McMILLAN, C.J.B.: Conceptos psicológicos en la educación. Ateneo, B. Aires.

HIRST, P.H. (1972): "Los aspectos lógico y psicológico de la enseñanza de un tema", eni PETERS, R.S. (ed): El concepto de educación. Paidós, B. Aires.

KNELLER, G.F. (1969): La lógica y el lenguaje en la educación. Ateneo, B. Aires.

KUETHE, J.L. (1971): Los procesos de enseñar y aprender. Paidós, B. Aires.

MAHONEY, M.J. (1974): “El procesamiento de información” en PEREZ GOMEZ, A. y ALMARAZ, J. (Comp.) (1982): Lecturas de aprendizaje y enseñanza. Zero, Madrid. MARIASJ. (1972): La estructura social. Revista de occidente, Madrid.

MARTIN, J.R. (1971): "sobre la reducción de 'saber que' a 'saber cómo"”, en SMITH, B.O. y ENNIS, R.H.: lenguaje y conceptos en educación. Ateneo, B. Aires.

NOVAK, J.D. (1982): Teoría y práctica de la educación. Alianza, Madrid.

ORTEGA Y GASSET, J. (1976): Ideas y creencias. Espasa Calpe, Madrid (8:a).

PASSMORE, J. (1983): Filosofía de la enseñanza. Fondo de cultura económica, México.

PEREZ GOMEZ, A. (1985): "Conocimiento académico y aprendizaje significativo. Bases teóricas para el diseño de instrucción", en GIMENO SACRISTAN, J. y PEREZ GOMEZ, A.: La enseñanza: su teoría y su práctica. Akal, Madrid.

SANVISENS MARFULL, A. (1983): "Concepción sistémico-cibernética de la educación" en VARIOS: Teoría de la educación I. (El problema de la educación). Límites, Murcia.

SARRAMONA LOPEZ, J. (1983): "La educación como sistema de comunicación". en VARIOS: Teoría de la educación I. Límites, Murcia.

WOODS, R.G. y BARROW, R.ST.C. (1978): Introducción a la filosofía de la educación. Anaya, Madrid. 